\title{
Selection of Reference Genes for Real-time Quantitative Polymerase Chain Reaction Analysis of Light-dependent Anthocyanin Biosynthesis in Chrysanthemum
}

\author{
Yan Hong and SiLan Dai ${ }^{1}$ \\ College of Landscape Architecture, Beijing Forestry University, Beijing 100083, China
}

\begin{abstract}
AdDitional INDEX wORDs. candidate gene, Chrysanthemum $\times$ morifolium, flower color, flower development, gene expression
Abstract. The light-dependent coloration of the vital organs of horticultural crops affects multiple parts of production and sales. The simplicity of the metabolic pathways of anthocyanins and the characteristics of lightdependent coloration make chrysanthemum (Chrysanthemum $\times$ morifolium) an ideal subject for studying the mechanism of light-regulated anthocyanin biosynthesis. In this study, real-time quantitative reverse transcriptionpolymerase chain reaction (PCR) was used in the analysis of the expression levels of anthocyanin biosynthesis genes in $C$. $\times$ morifolium 'Reagan'. The reference genes selected were those assumed to remain at constant levels in three flower color lines at five floral developmental stages and at two light conditions. Using digital gene expression technology, we selected nine reference genes with moderate expression in the chrysanthemum ray florets at various floral developmental stages under illuminated and dark conditions as the candidate reference genes for further study. After comprehensively analyzing the stability of gene expression with three distinct statistical algorithms, geNorm, NormFinder, and qBase plus, we found that $F$-box and $P P 2 A$ were the most stable genes in all of the samples. In addition, we analyzed the relative expression level of the $\mathrm{CmF3H}$ gene in different samples to verify the reference genes that we selected. This study provides a consensus list of validated reference genes that will benefit future studies of the expression of chrysanthemum genes involved in anthocyanin biosynthesis and floral development under various light conditions. Moreover, this information will also promote the molecular breeding of horticultural crops for their color modification.
\end{abstract}

The fruits and flower color of agricultural products are very important for their commercial value. Anthocyanin, one of the key substances that makes plant organs present different colors, is coregulated by the development of the plant itself and environmental factors (Tanaka and Ohmiya, 2008). Of these environmental factors, light is one of the most important factors to affect the biosynthesis of anthocyanin (Albert et al., 2009). As a result, a large number of studies on the mechanisms of light-regulated anthocyanin biosynthesis has been reported in many species. In general, the mechanisms showed significant species specificity that can be divided into the following three types according to the different modes of light regulation: the light-dependent type (Dong et al., 1998), the partial lightdependent type (Zhou, 2004), and the light-independent type (Xu and Li, 2006). Accordingly, two hypotheses pertaining to the light-dependent and light-independent anthocyanin biosynthetic pathways have been proposed and tested in many crops. For instance, the coloration of grapes (Vitis vinifera) grown under light conditions showed species specificity (Zheng et al., 2013). The expression levels of anthocyanin biosynthetic genes in red chinese sand pear (Pyrus pyrifolia) were affected by different organs, cultivars, bagging treatments, and fruit sides (Yu et al., 2012). In apple (Malus pumila), the interaction

Received for publication 22 Oct. 2014. Accepted for publication 17 Nov. 2014. This work was supported by the Project of Introducing International Advanced Forestry Science and Technology of National Forestry Administration (No. 2013-428 ) and the National Natural Science Foundation of China (Grant No. 31272192). We thank our colleagues in the chrysanthemum breeding group for their valuable contributions.

${ }^{1}$ Corresponding author. E-mail: silandai@sina.com. between MdCOP1 ubiquitin E3 ligases and the MdMYB1 gene regulated the light-induced anthocyanin biosynthesis and red fruit color (Li et al., 2012). Although some progress has been made, the molecular mechanism of light-dependent anthocyanin biosynthesis is still unclear, which restricts the molecular breeding of horticultural crops for their color modification (Zheng et al., 2013).

Chrysanthemum is an ornamental crop with worldwide fame, and the yield, output value, and rich germplasm make it a leader in the global flower industry (Anderson, 2006). Sun et al. (2010) reported that in chrysanthemum anthocyanin metabolism, there is only the cyanidin metabolic pathway. The simple background of pigment metabolism makes chrysanthemum an ideal subject for studying anthocyanin biosynthesis and its molecular regulatory mechanisms that respond to environmental factors such as light and temperature. Gene profiling or expression analysis provides improved understanding and insight into the complex regulatory networks and mechanisms of the genetic, signaling, and metabolic pathways that underlie the developmental, biological, and cellular processes (Yeap et al., 2014). Several studies to reveal the molecular mechanisms behind the generation of flower color in chrysanthemum have been reported (Brugliera et al., 2013; Huang et al., 2013; Noda et al., 2013; Zhao et al., 2013). Therefore, knowing the expression patterns of key genes involved in the anthocyanin biosynthetic pathway of chrysanthemum under various environmental conditions will help us to understand the molecular mechanism of color generation by anthocyanin.

With the rapid development of molecular biology, several methods for detecting transcript abundance of genes in plant studies have been developed, including Northern blotting, in 
situ hybridization and real-time quantitative reverse transcriptionPCR [RT-qPCR (Higuchi et al., 1993)]. Of these biological techniques, RT-qPCR is currently regarded as the most reliable method for detecting and measuring low amounts of mRNAs as a result of its sensitivity, accuracy, specificity, and high throughput (Artico et al., 2010; Bustin, 2002; Nolan et al., 2006). RT-qPCR provides clear advantages in quantifying biologically relevant changes in mRNA levels. However, the accuracy of the method is influenced by variables such as the genotype of the samples, variability in the RNA obtained from different tissues or tissue treatments, variability in reverse transcription, and amplification efficiencies of primers (Chao et al., 2012). Therefore, it is vital to select ideal reference genes to normalize RT-qPCR analysis. Recently, research into evaluating the stability of potential reference genes across various experimental conditions has been performed in different plant species such as chamomile [Chrysanthemum lavandulifolium (Fu et al., 2013)], cineraria [Senecio cruentus (Jin et al., 2013)], blueberry [Vaccinium vitis (Die and Rowland, 2013)], oil palm [Elaeis quineensis (Yeap et al., 2014)], chinese iris [Iris lactea var. chinensis (Gu et al., 2014)], and mei flower [Prunus mume (Wang et al., 2014)]. These studies have revealed that the expression of commonly used reference genes may vary considerably in different species, organs, or under different developmental stages and experimental conditions. Nevertheless, little information, if any, is available concerning the reference genes for gene expression analyses of light-dependent anthocyanin biosynthesis and floral development in chrysanthemum.

With the availability of plant genome and transcriptome sequences, transcriptomic libraries have been widely applied in the selection of candidate reference genes as previously reported in tomato [Solanum lycopersicum (Coker and Davies, 2003)], perennial ryegrass [Lolium perenne (Lee et al., 2010)], and arabidopsis [Arabidopsis thaliana (Czechowski et al., 2005)]. In the present study, digital gene expression (DGE) technology was used to analyze the differences in gene expression between the illuminated and non-illuminated transcriptomic libraries of chrysanthemum generated in our preliminary study (data not shown). Nine candidate reference genes with little variation between the two libraries, including three traditional reference genes and six new ones, were selected for further study. We then compared the performance of these nine candidate reference genes in the $C$. ×morifolium 'Reagan' samples of three color lines at five developmental stages and under two different light conditions. Three distinct statistical algorithms, geNorm (Vandesompele et al., 2002), NormFinder (Andersen et al., 2004), and qBase plus (Hellemans et al., 2007), were used to determine the most suitable reference genes for the normalization of gene expression in chrysanthemum. Finally, to verify the usefulness of the selected reference genes, the expression pattern of $\mathrm{CmF3H}$, one of the key genes in the anthocyanin biosynthetic pathway, was analyzed in all samples. This study provides a consensus list of validated reference genes that will benefit future studies of the expression of anthocyanin biosynthesis genes and floral development genes under various light conditions in chrysanthemum. Moreover, these findings will also promote the molecular breeding of horticultural crops for their color modification.

\section{Materials and Methods}

Plant material and treatments. In the chrysanthemum cultivar Reagan, the anthocyanin biosynthesis in the ray florets of the capitulum is light-dependent. Thus, the capitula from three color lines (purple, red, and pink) were selected as the plant material in this study. During the development of the capitulum, a total of six stages were defined: S0, S1, S2, S3, S4, and S5 (Sun et al., 2010). All samples were collected in Sept. 2013 from a growth chamber (the intensity of illumination was $50 \pm 5 \mu \mathrm{mol} \cdot \mathrm{m}^{-2} \cdot \mathrm{s}^{-1}$, the photoperiod was $16 \mathrm{~h}$ in the daytime and $8 \mathrm{~h}$ at night, the temperature was $20 \pm 1{ }^{\circ} \mathrm{C}$, and relative humidity was $60 \%$ ) located at the Beijing Forestry University, Beijing, China. To investigate the effects of light on anthocyanin biosynthesis during floral development, two light treatments (illuminated condition and dark condition) were used on the five floral developmental stages. These stages were sampled as follows: L1, L2, L3, L4, and L5 were treated under a fluorescent lamp and were sampled during the capitulum developmental stages S1, S2, S3, S4, and S5, respectively; D1, D2, D3, D4, and D5 were 100\% shaded using silver papers at the S0 stage and were sampled once every other $48 \mathrm{~h}$ when they developed to the S1, S2, S3, S4, and S5 stages, respectively (Fig. 1; Supplemental Table 1). All of the samples were transported to the laboratory immediately after acquisition and were then frozen in liquid nitrogen and stored at $-80^{\circ} \mathrm{C}$.

RNA EXTraction, QUALity Controls, DNASE I digestion, AND FIRST STRAND CDNA SYNTHESIS. Total RNA was isolated using a Quick RNA Isolation Kit (Huayueyang Biotechnology Co., Beijing, China) from the ray florets of the three color lines at different floral developmental stages and light conditions. RNA quality was assessed by combining information from several control steps. First, the purity was inferred from the absorption ratios using a spectrophotometer (NanoDrop ND-1000; Thermo Fisher Scientific, Waltham, MA). Only the RNA samples with an $\mathrm{A}_{260} / \mathrm{A}_{280}$ ratio between 1.9 and 2.1 and an $\mathrm{A}_{260} / \mathrm{A}_{230}$ ratio greater than 2.0 were used for further analysis. Then, the integrity of the RNA samples was visualized using $1 \%(\mathrm{w} / \mathrm{v})$ agarose gel electrophoresis stained with ethidium bromide. Finally, all RNA samples were pretreated with RNase-free DNase I (Promega, Madison, WI) at $37^{\circ} \mathrm{C}$ for $30 \mathrm{~min}$ to eliminate any DNA contamination. The first strand cDNA was synthesized from $1 \mu \mathrm{g}$ of total RNA using the M-MLV reverse transcription system (Promega) according to the manufacturer's protocol.

SEleCtion OF CANDIDATE REFERENCE GENES. In our preliminary study, five Illumina HiSeq transcriptomic libraries of C. Xmorifolium 'Reagan' were constructed. In total, 103,517 unigenes were identified, of which 60,712 were annotated against four public protein databases (data not shown). Using the DGE technology, the relative expression levels of these unigenes were analyzed in the ray florets of the illuminated L1, L2, and L3 samples and the non-illuminated D2 and D3 samples. The gene expression level was calculated using the fragments per kilobase per million fragments (FPKM) values (Mortazavi et al., 2008). The difference in gene expression between the samples was determined based on the FPKM value. As many as 25,006 unigenes showed little variation in the DGE technology. From these unigenes, nine reference genes that showed the most stable expression $\left(\mid \log _{2}\right.$ ratio $\left.\mid \leq 0.5\right)$ were selected as candidates for the normalization of gene expression, including actin-related protein (Actin), elongation factor $1 \alpha(E f l \alpha)$, beta-tubulin ( $\beta$-tubulin), calcium-dependent protein kinase $(C D P K), \mathrm{ABC}$ transporter $(A B C)$, protein phosphatase 2A $(P P 2 A)$, F-box protein $(F-b o x)$, E3 ubiquitin-protein ligase (E3), and TIP41-like protein (TIP41). These genes were evaluated in three color lines of C. ×morifolium 'Reagan' at five floral developmental stages 


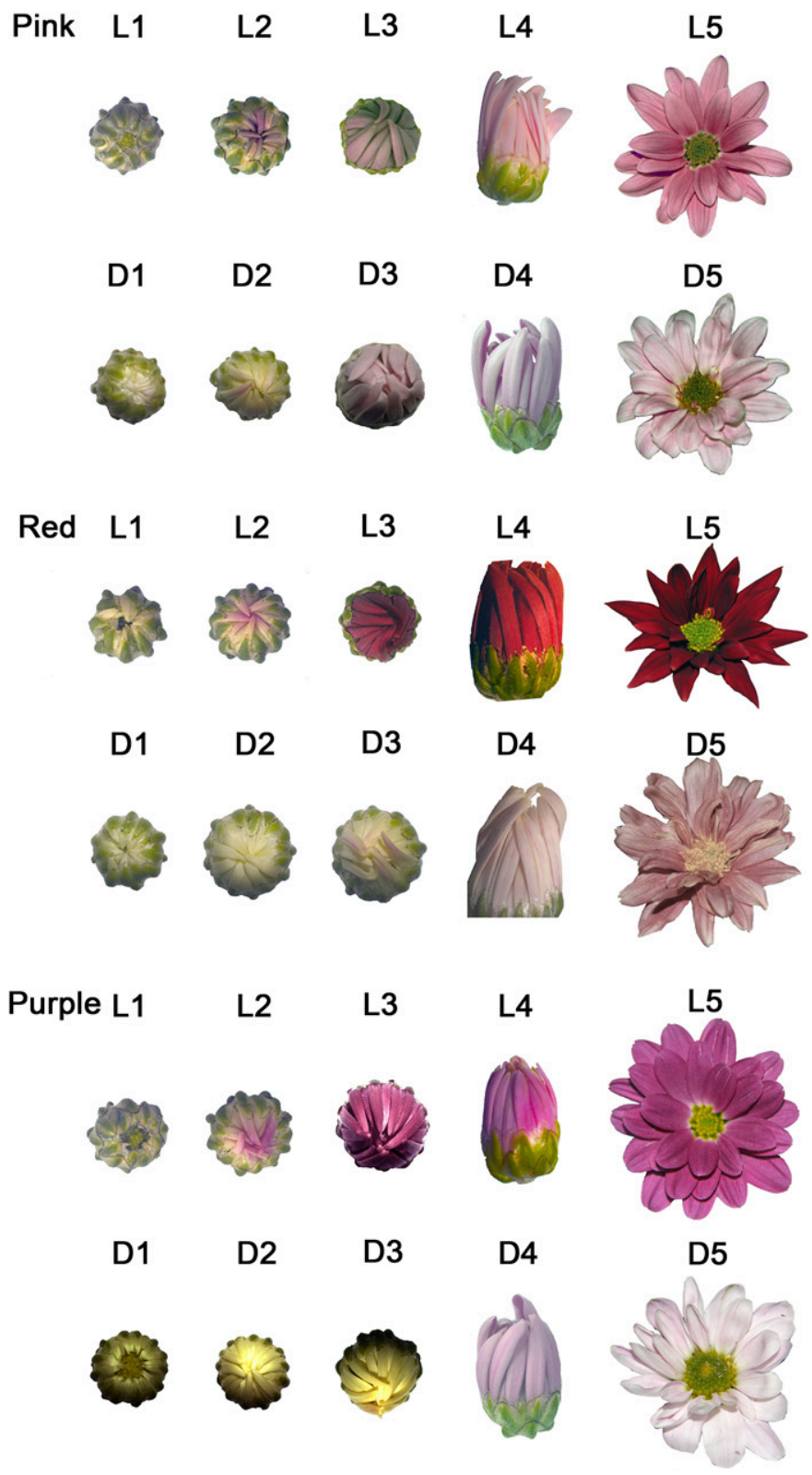

Fig. 1. All chrysanthemum samples used in the present study. L1, L2, L3, L4, and L5 were samples treated under a fluorescent lamp and were sampled during the capitulum developmental stages S1, S2, S3, S4, and S5, respectively; D1, D2, D3, D4, and D5 were 100\% shaded using silver papers at the S0 stage and were sampled once every other $48 \mathrm{~h}$ when they developed to the S1, S2, S3, S4, and S5 stages, respectively. Pink, red, and purple refer to different color lines within the chrysanthemum cultivar Regan.

and under two light conditions. Among these genes, Actin, $\beta$-tubulin, and E3 were traditional reference genes, whereas the other six were new genes we selected (Table 1).

Primer Design AND TeSt OF AMPLIFICATION EFFiCIENCY. Primers were designed using Primer 3 software (Rozen and Skaletsky, 1999) with the following criteria: melting temperature of 55 to $70{ }^{\circ} \mathrm{C}$, primer lengths of 20 to $25 \mathrm{bp}$, and amplicon lengths of 100 to $300 \mathrm{bp}$ (Table 1). A melting curve analysis was performed by RT-qPCR after 40 cycles of amplification. The specificity of the primer amplicons was verified by $2 \%(\mathrm{w} / \mathrm{v})$ gel electrophoresis.

RT-QPCR ANALYSIS. PCR reactions were performed using a Mini Opticon Real-time PCR System (Bio-Rad, Hercules,
CA) based on the SYBR Premix Ex Taq (Takara Bio, Shiga, Japan). Reactions were prepared in a total volume of $20 \mu \mathrm{L}$ containing $2 \mu \mathrm{L}$ of template $(\approx 50 \mathrm{ng}), 0.4 \mu \mathrm{L}$ of each amplification primer $(10 \mu \mathrm{mol}), 10 \mu \mathrm{L}$ of $2 \times$ SYBR Premix Ex Taq, and 7.2 $\mu \mathrm{L}$ of ddH2O. The following standard thermal profile was used for all PCR reactions: $95^{\circ} \mathrm{C}$ for $30 \mathrm{~s}$ followed by 40 cycles of $95^{\circ} \mathrm{C}$ for $5 \mathrm{~s}$, the optimal annealing temperature for $30 \mathrm{~s}$, and $72{ }^{\circ} \mathrm{C}$ for $30 \mathrm{~s}$. Melting curves were recorded after 40 cycles by heating from 65 to $95{ }^{\circ} \mathrm{C}$ by increasing the temperature stepwise by $0.5{ }^{\circ} \mathrm{C}$ every $5 \mathrm{~s}$. PCR amplification efficiency was derived from a standard curve generated by a 10fold serial dilution of cDNA. Negative controls were included for each primer pair and each RT-qPCR reaction was performed in triplicate.

Data Analysis. The expression levels of the tested reference genes were determined by quantification cycle $(\mathrm{Cq})$ values, in which the quantification of the fluorescence reached a specific threshold level of detection. The $\mathrm{Cq}$ values were converted to relative values with the lowest $\mathrm{Cq}$ value set to one and the amplification efficiencies for each primer pair were incorporated (Table 1). To determine which reference genes were best suited for transcript normalization in chrysanthemum, the data obtained for each sample were analyzed using the geNorm [Version 3.4 (Vandesompele et al., 2002)], NormFinder [Version 0.953 (Andersen et al., 2004)], and qBase plus (Hellemans et al., 2007) programs according to their respective protocols. These strategies provided complementary measures of gene expression stability among the cDNA samples.

Assessment of nORMAlization. $\mathrm{CmF3H}$ was previously cloned by the authors and was used as a target gene to demonstrate the reliability of the results based on the set of reference genes identified here. The expression levels of $\mathrm{CmF3H}$ in different samples were quantified using stable reference gene(s) or a combination of reference genes determined by geNorm. Primer pairs of forward (5'GGTGCAAGATTGGA GGGAGAT3') and reverse (5'CAGGTTGAGGACAC TTTGGGTAG3') for $\mathrm{CmF3H}$ were used.

\section{Results}

Performance of the PRIMers for each Reference gene. The results of the agarose gel electrophoresis demonstrated that all nine primer pairs amplified a single band of the expected size from various cDNA templates. The correlation coefficient $\left(R^{2}\right)$ indicated the quality of the fit of the standard curve to the plotted data points. The $R^{2}$ values of all of the primer pairs were greater than 0.9800 and ranged between $0.9800(A B C)$ and 0.9990 ( $\beta$-tubulin), indicating a strong relationship between the detected $\mathrm{Cq}$ values and the corresponding relative amount of template in all of the amplification reactions (Table 1). PCR amplification efficiency indicated the amplicon doubling rates of specific primer pairs during amplification, which ranged from $90.5 \%$ (F-box) to $107.9 \%(P P 2 A)$ and were obtained from the standard curves generated using a 10-fold serial dilution of the cDNA used in this study (Table 1). These results reflected the high quality and specificity of the RT-qPCR reactions. Therefore, these candidate genes were used in the next assay.

EXPRESSION PROFILES OF CANDIDATE REFERENCE GENES. The $\mathrm{Cq}$ values for the nine genes were pooled so that the expression levels of each gene could be compared. The expression profiles of the nine candidate reference genes presented as $\mathrm{Cq}$ values are shown in Figure 2. The mean values of the reference genes were 


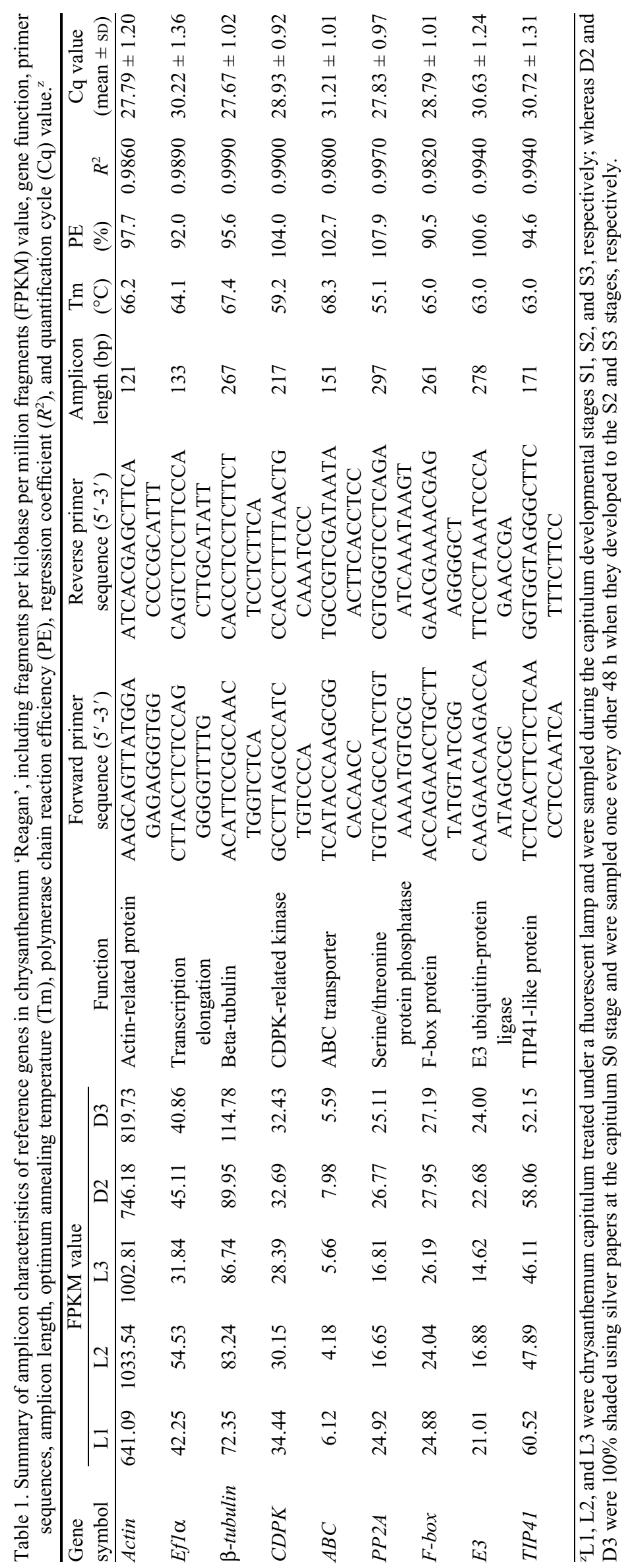




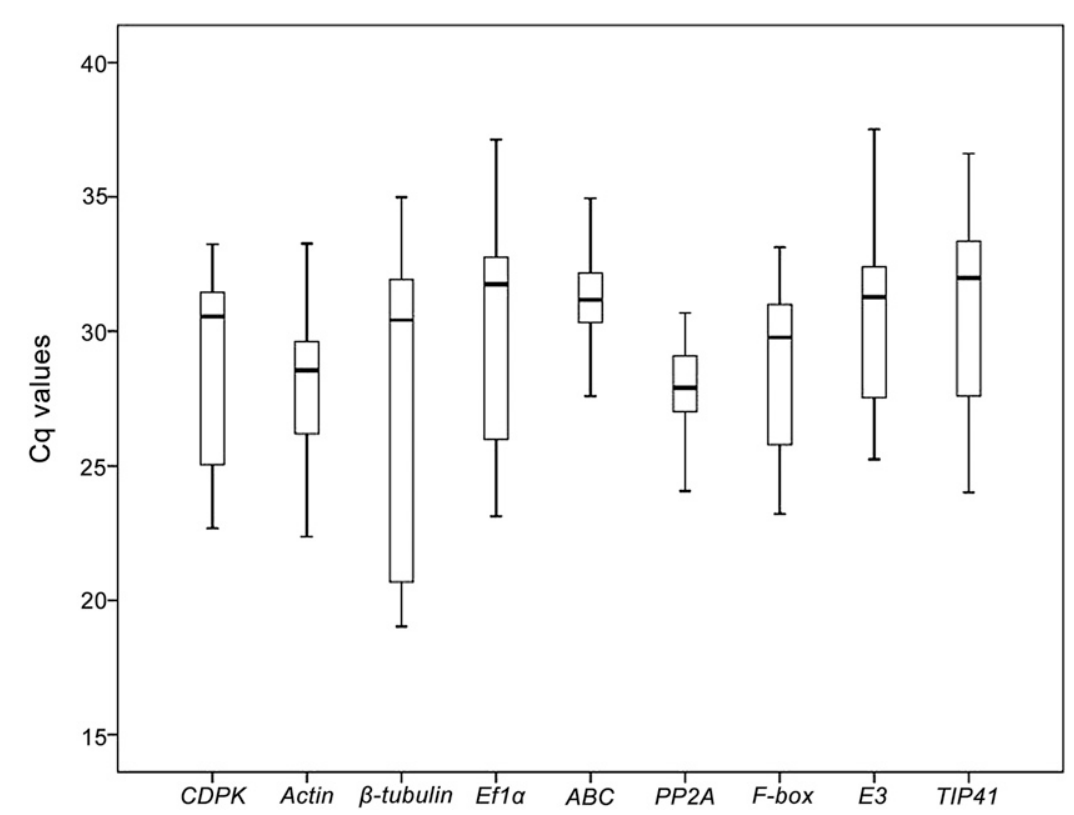

Fig. 2. Mean quantification cycle $(\mathrm{Cq})$ values of nine candidate reference genes. The data were pooled from all samples. Boxes indicate the $25 \mathrm{th} / 75$ th percentiles, the line marks the median, squares represent the means, and whiskers indicate the ranges for the total samples.

$P P 2 A$ were the most stable genes under all of the experimental conditions.

Another important analysis performed using geNorm was the determination of the optimal number of reference genes or the effects of adding extra genes to the analysis by calculating the pairwise variation (V) between two sequential candidate genes. A $V_{n} / V_{n+1}$ value lower than 0.015 under all experimental conditions indicates that adding an extra gene to obtain a reliable normalization factor (NF) was unnecessary. Based on this parameter, the combination of three reference genes, F-box, TIP41, and CDPK, were recommended for all samples because the pairwise variation value was lower than 0.015 (Fig. 3B). Two reference genes were sufficient for obtaining accurate results in the different flower color lines and illuminated samples because the $V_{2 / 3}$ values were lower than or equal to 0.015 . Thus, the combinations of F-box and PP2A or F-box and TIP4I were the best reference selections in these four samples. For the non-illuminated treatment, the $V_{4 / 5}$ value was lower than 0.015 , so four reference genes were chosen for

between 27.67 ( $\beta$-tubulin) and $31.21(A B C)$, which represented the different expression levels in $C$. Xmorifolium 'Reagan'. $\beta$-tubulin showed the highest expression level in all samples and the lowest $\mathrm{Cq}$ value (19.03). E3 presented the lowest expression level and the highest Cq value (37.52). Generally, the expression levels of most genes ranged from 27.89 to 31.75 , and the $\mathrm{Cq}$ values of all candidate genes were dispersive, which indicated that none of the selected genes was expressed stable in the different samples (Fig. 2). Therefore, it was necessary to evaluate the reference genes for normalization under different experimental conditions.

The STABility of CANDidate RefERence Genes. In the present study, three methods were selected for analyzing the expression stability of genes. The first method, geNorm, calculates the average expression stability (M) based on the average pairwise variation between all genes tested. A lower $M$ value indicates a more stable gene expression, whereas a higher M value indicates less stable gene expression. Stably expressed genes have values below 1.5 (Vandesompele et al., 2002) or 0.5 (Hellemans et al., 2007). The ranking order of genes used in this study according to their $M$ values is depicted in Figure 3A, and the $\mathrm{M}$ values for all genes were far lower than 0.5 . Because only the ray floret was considered in the present study, a lower threshold of 0.12 was adopted. $F$-box and TIP 41 were the most stably expressed genes with M values of $0.0907 ; \beta$-tubulin and $A B C$ were the least stable genes in all samples (Fig. 3A). In different flower color lines across various floral developmental stages, $F$-box and $P P 2 A$ all performed well with $\mathrm{M}$ values of $0.0889,0.0844$, and 0.0839 in the purple, pink, and red lines, respectively, whereas Efl $\alpha$ and Actin were the least stable genes in the purple line with the respective $\mathrm{M}$ values of 0.1706 and 0.1674. TIP41,Efl $\alpha$, and $E 3$ had unstable expression patterns in the pink and red lines. For the illuminated and nonilluminated samples, F-box and TIP41 showed the same performance as in the total samples. Generally, F-box and normalizing the expression pattern results of the target genes (Fig. 3B).

The stability of reference genes was reanalyzed using NormFinder to confirm the results obtained with geNorm. NormFinder is based on variance estimation and takes intragroup and intergroup variations into account in calculating the NF. Genes were then ranked according to their stability under a given set of experimental conditions. In our study, the ranking order generated by this method was slightly different from the results obtained with geNorm (Table 2). F-box was still the most stable gene in all of the tested samples, whereas $C D P K$ ranked second using this method and was more stable than TIP41. In different flower color lines at various floral developmental stages, the two most stable genes calculated by NormFinder were the same as those determined by geNorm, but according to the NormFinder results, $A B C$ and $C D P K$ were more stable than $F$-box in the pink and red lines, respectively. For samples under different light conditions, F-box and TIP4l were still the most stable genes under illuminated conditions, whereas in the non-illuminated samples, Actin was more stable than TIP41 (Table 2).

Finally, the Cq values and specific amplification efficiencies for each candidate reference gene were inputted into qBase plus to further evaluate their expression stabilities. qBase converts the data into normalized relative quantities. In this study, F-box and TIP41 were identified as the most stable reference genes in all samples and under different light conditions, as shown in Table 3. F-box and PP2A also performed well in the different flower color lines with the same stabilities as those determined by geNorm.

REFERENCE GENE VALIDATION. F3H leads to the formation of dihydroflavonols, which is a required substance for anthocyanin biosynthesis. To verify the reliability of the selected reference genes, we used four normalization approaches according to the results of geNorm to analyze the expression 


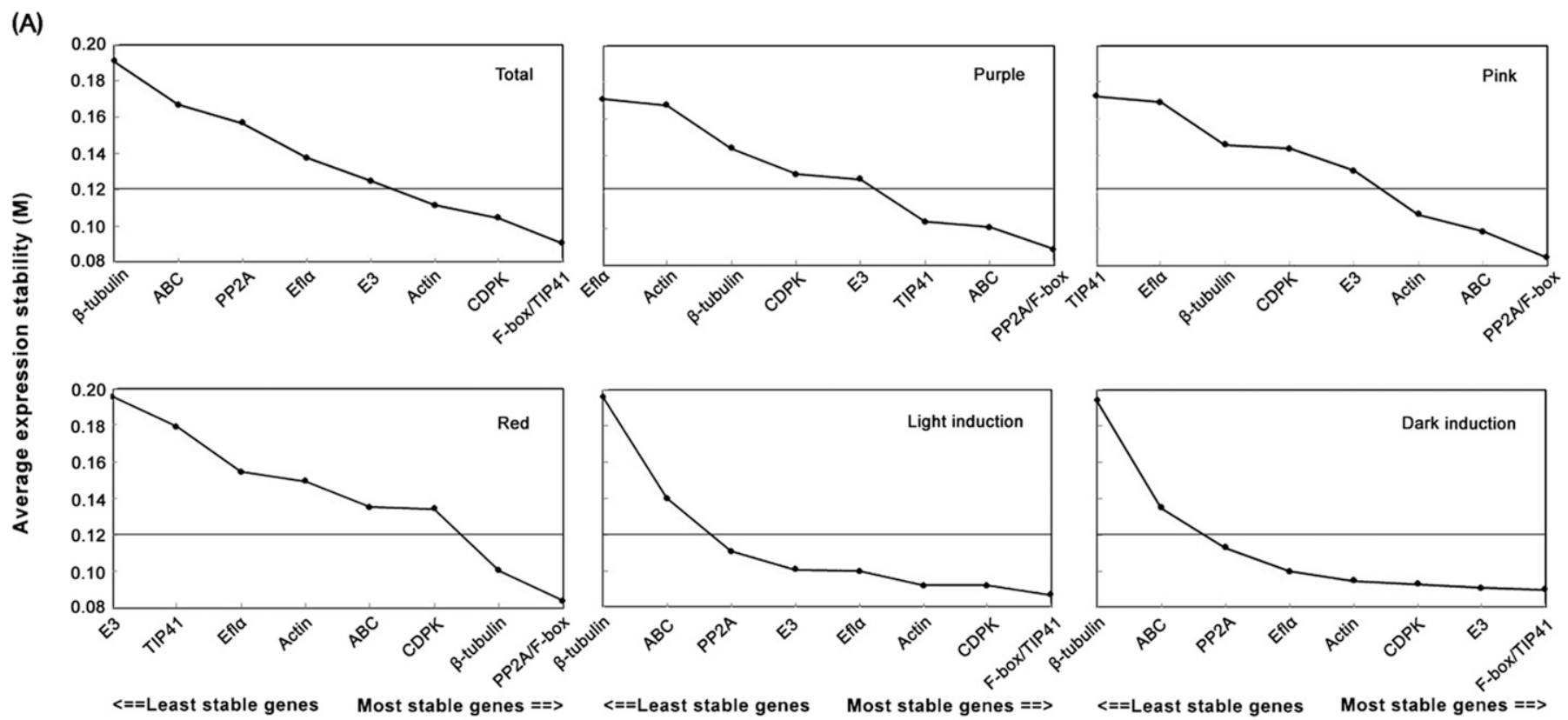

(B)

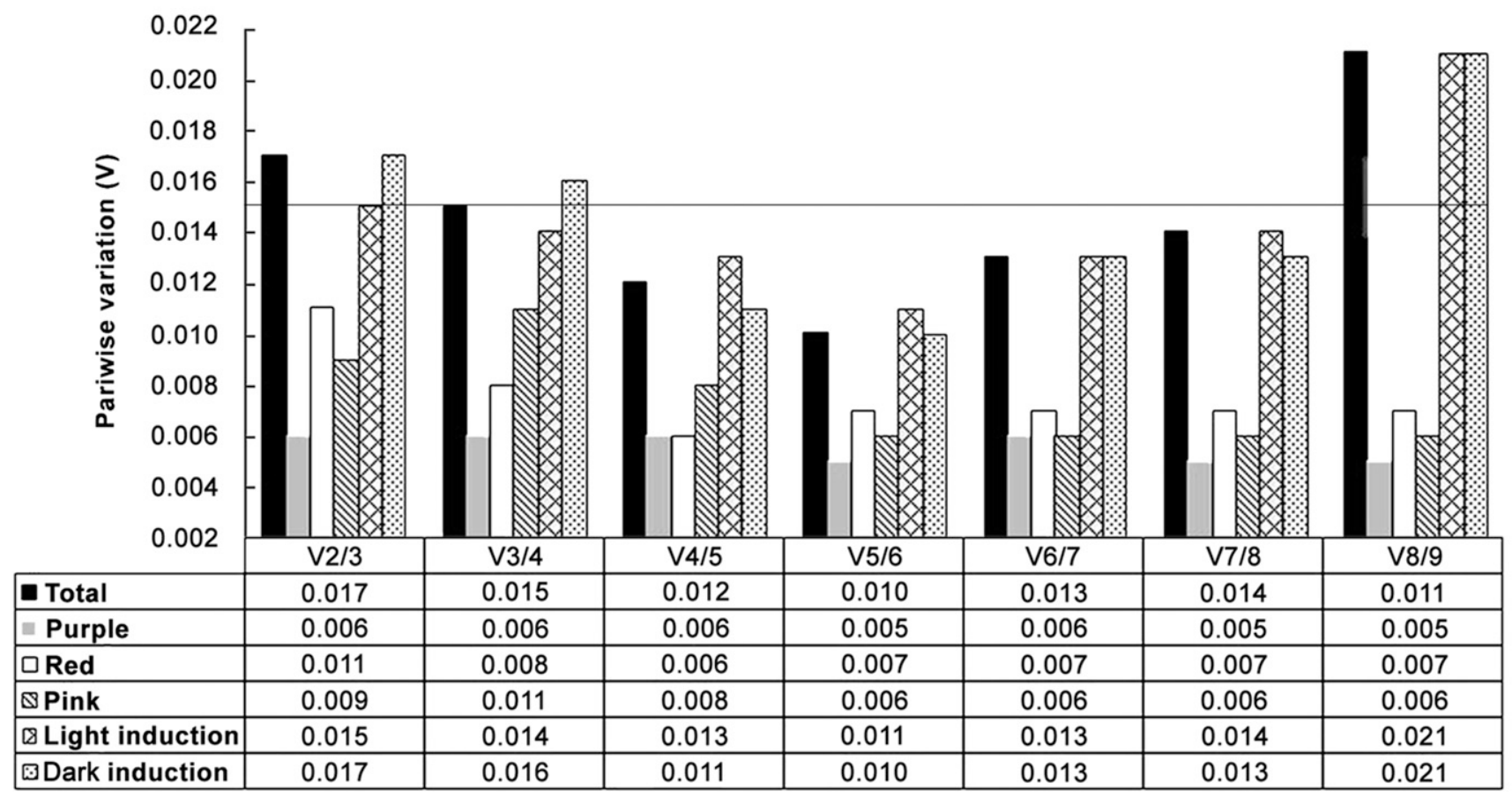

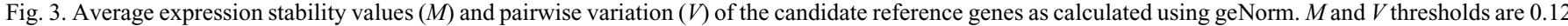
and 0.015 , respectively. (A) Average expression stability values after stepwise exclusion of the least stable genes across all treatment groups. The least stable genes are on the left and the most stable genes are on the right; (B) the optimal number of reference genes required for effective normalization. The pairwise variation $\left(V_{n} / V_{n+1}\right)$ was analyzed between the normalization factors $\mathrm{NF}_{\mathrm{n}}$ and $\mathrm{NF}_{\mathrm{n}+1}$.

patterns of $\mathrm{CmF} 3 \mathrm{H}$ during different floral developmental stages of the three flower color lines under different light conditions. When normalized using the most stable reference genes $F$-box or $P P 2 A$, either singly or in combination, the expression patterns of $\mathrm{CmF3H}$ were almost identical with only slight differences (Fig. 4A and B). In contrast, when normalized using the least stable gene, $\beta$-tubulin, the expression pattern showed significant differences. The highest expression level was different when the least and most stable reference genes were used for normalization (Fig. 4A and B). The expression level of
$\mathrm{CmF3H}$ was highest in the S3 stage when normalized to the least stable gene but was highest in the S5 stage when normalized to the two most stable reference genes either separately or in combination. Interestingly, the expression level of $\mathrm{CmF3H}$ increased with the floral development under the two light conditions. Compared with the illuminated samples, the expression levels of $\mathrm{CmF3H}$ in the non-illuminated samples were significantly lower during each floral developmental stage, indicating that the expression of $\mathrm{CmF3H}$ was repressed after shading. 
Table 2. Ranking of reference genes and their expression stability values calculated using NormFinder. ${ }^{\mathrm{z}}$

\begin{tabular}{|c|c|c|c|c|c|c|c|c|c|c|c|c|}
\hline \multirow{2}{*}{$\begin{array}{l}\text { Ranking } \\
\text { order }\end{array}$} & \multicolumn{2}{|c|}{ Total } & \multicolumn{2}{|c|}{ Purple } & \multicolumn{2}{|c|}{ Pink } & \multicolumn{2}{|c|}{ Red } & \multicolumn{2}{|c|}{ Illuminated condition } & \multicolumn{2}{|c|}{ Dark condition } \\
\hline & Gene & Stability & Gene & Stability & Gene & Stability & Gene & Stability & Gene & Stability & Gene & Stability \\
\hline$\overline{1}$ & $F$-box & 0.129 & F-box & 0.297 & $P P 2 A$ & 0.393 & $P P 2 A$ & 0.297 & F-box & 0.004 & F-box & 0.004 \\
\hline 2 & $C D P K$ & 0.230 & $P P 2 A$ & 0.304 & $A B C$ & 0.455 & $C D P K$ & 0.551 & TIP41 & 0.005 & Actin & 0.007 \\
\hline 4 & TIP41 & 0.271 & $A B C$ & 0.416 & Actin & 0.622 & F-box & 0.588 & $C D P K$ & 0.008 & $E f 1 \alpha$ & 0.009 \\
\hline 5 & $E 3$ & 0.358 & TIP41 & 0.454 & $C D P K$ & 0.745 & Actin & 0.625 & $E f 1 \alpha$ & 0.011 & TIP41 & 0.009 \\
\hline 6 & $E f l \alpha$ & 0.406 & $C D P K$ & 0.476 & E3 & 0.767 & $\beta$-tubulin & 0.677 & E3 & 0.013 & $C D P K$ & 0.009 \\
\hline 8 & $A B C$ & 0.836 & Actin & 0.611 & $E f 1 \alpha$ & 0.882 & $E f 1 \alpha$ & 0.913 & $A B C$ & 0.025 & $A B C$ & 0.023 \\
\hline 9 & $\beta$-tubulin & 1.082 & $E f 1 \alpha$ & 0.634 & TIP41 & 1.021 & $E 3$ & 0.940 & $\beta$-tubulin & 0.030 & $\beta$-tubulin & 0.031 \\
\hline
\end{tabular}

${ }^{\mathrm{z}}$ Total, purple, pink, red, illuminated condition and dark condition represent all samples, samples of purple color line, pink color line, red color line, samples treated under fluorescent lamp, and dark condition, respectively.

Table 3. Ranking of reference genes and the mean CV values calculated using qBase plus. ${ }^{\mathrm{z}}$

\begin{tabular}{|c|c|c|c|c|c|c|c|c|c|c|c|c|}
\hline \multirow{2}{*}{$\begin{array}{l}\text { Ranking } \\
\text { order }\end{array}$} & \multicolumn{2}{|c|}{ Total } & \multicolumn{2}{|c|}{ Purple } & \multicolumn{2}{|c|}{ Pink } & \multicolumn{2}{|c|}{ Red } & \multicolumn{2}{|c|}{ Illuminated condition } & \multicolumn{2}{|c|}{ Dark condition } \\
\hline & Gene & $\mathrm{CV}$ & Gene & $\mathrm{CV}$ & Gene & $\mathrm{CV}$ & Gene & $\mathrm{CV}$ & Gene & $\mathrm{CV}$ & Gene & $\mathrm{CV}$ \\
\hline$\overline{1}$ & F-box & 0.129 & F-box & 0.143 & $P P 2 A$ & 0.133 & F-box & 0.146 & F-box & 0.147 & F-box & 0.139 \\
\hline 2 & TIP41 & 0.352 & $P P 2 A$ & 0.285 & F-box & 0.210 & $P P 2 A$ & 0.293 & TIP41 & 0.385 & TIP41 & 0.254 \\
\hline 4 & $C D P K$ & 0.466 & $A B C$ & 0.449 & Actin & 0.374 & $C D P K$ & 0.380 & $C D P K$ & 0.523 & $P P 2 A$ & 0.497 \\
\hline 5 & $E f 1 \alpha$ & 0.732 & Actin & 0.521 & $A B C$ & 0.521 & Actin & 0.471 & Actin & 0.674 & E3 & 0.503 \\
\hline 6 & $E 3$ & 0.855 & $C D P K$ & 0.582 & TIP41 & 0.732 & $\beta$-tubulin & 0.528 & E3 & 0.952 & Actin & 0.642 \\
\hline 8 & $A B C$ & 1.128 & E3 & 0.738 & $E f 1 \alpha$ & 0.896 & $E f l \alpha$ & 0.732 & $A B C$ & 1.251 & $A B C$ & 0.938 \\
\hline 9 & $\beta$-tubulin & 1.206 & $E f l \alpha$ & 0.926 & E3 & 0.937 & $E 3$ & 0.851 & $\beta$-tubulin & 1.294 & $\beta$-tubulin & 1.027 \\
\hline
\end{tabular}

${ }^{\mathrm{z} T o t a l}$, purple, pink, red, illuminated condition. and dark condition represent all samples, samples of purple color line, pink color line, red color line, samples treated under fluorescent lamp. and dark condition, respectively.

\section{Discussion}

THE SIGNIFICANCE OF SYSTEMATIC VERIFICATION FOR MULTIPLE REFERENCE GENES. Ideal reference genes should be stably expressed under all experimental conditions and independent of organs, tissues, developmental stages, and different treatments (Huggett et al., 2005). Thus, the use of one or more stable reference genes in RT-qPCR has progressed in tandem with the evolution of mathematical equations for quantification, moving from the one-reference model (Livak and Schmittgen, 2001; Pfaffl, 2001) to modern advanced relative quantification models with multiple reference genes (Hellemans et al., 2007). Therefore, a systematic verification for multiple reference genes under specific experimental conditions is extremely important for gene expression studies using RT-qPCR (Fu et al., 2013).

THE HIGH EFFICIENCY OF CANDIDATE REFERENCE GENE SELECTION USING TRANSCRIPTOMIC LIBRARIES. The frequently used methods for candidate reference gene selection are literature review and searching public databases of expressed sequence tags for reference genes related to certain biological processes such as glycolysis, cellular metabolism, protein synthesis, and degradation (Yeap et al., 2014). However, traditional reference genes are usually obtained using these methods. Several studies have showed that the expression of traditional reference genes might be unstable under different experimental conditions (Gutierrez et al., 2008), which might affect the expression analysis of target genes to a large extent. For instance, Gutierrez et al. (2008) found that compared with those validated reference genes, the normalization of target gene expression showed a 100 -fold deviation using those invalidated reference genes. Using traditional reference genes blindly may result in wrong or even opposite results (Huggett et al., 2005; Vandesompele et al., 2002).

The transcriptome is a necessary link that connects genetic information and functional proteins (Martin and Wang, 2011), and its characteristics of high throughput and efficiency make it an important method for gene expression studies (Lee et al., 2010). Through the transcriptome approach, sequence information for thousands of genes can be analyzed simultaneously, quickly, and accurately. Moreover, based on transcriptomic libraries, the expression of thousands of genes can be detected rapidly using DGE technology (Martin and Wang, 2011). Through the analysis of transcriptomic data, the stable reference genes expressed in different cultivars, organs, and under various experimental conditions can thus be screened out efficiently. This approach has been successfully applied in many studies of plants such as tomato (Coker and Davies, 2003), perennial ryegrass (Lee et al., 2010), and arabidopsis (Czechowski et al., 2005). In the present study, we used DGE technology to screen out nine candidate chrysanthemum reference genes with moderate expression in five transcriptomic libraries. Of these, six were new genes, laying a foundation for the comprehensive evaluation of the expression stability of these candidate reference genes.

THE STABILITY OF CHRYSANTHEMUM REFERENCE GENES USED IN THE EXPRESSION ANALYSIS OF ANTHOCYANIN BIOSYNTHESIS GENES AND FLORAL DEVELOPMENT GENES. Chrysanthemum is an ideal crop for anthocyanin research because in chrysanthemum, only the cyanidin metabolic pathway is related to the anthocyanin metabolism and the background of pigment metabolism is 

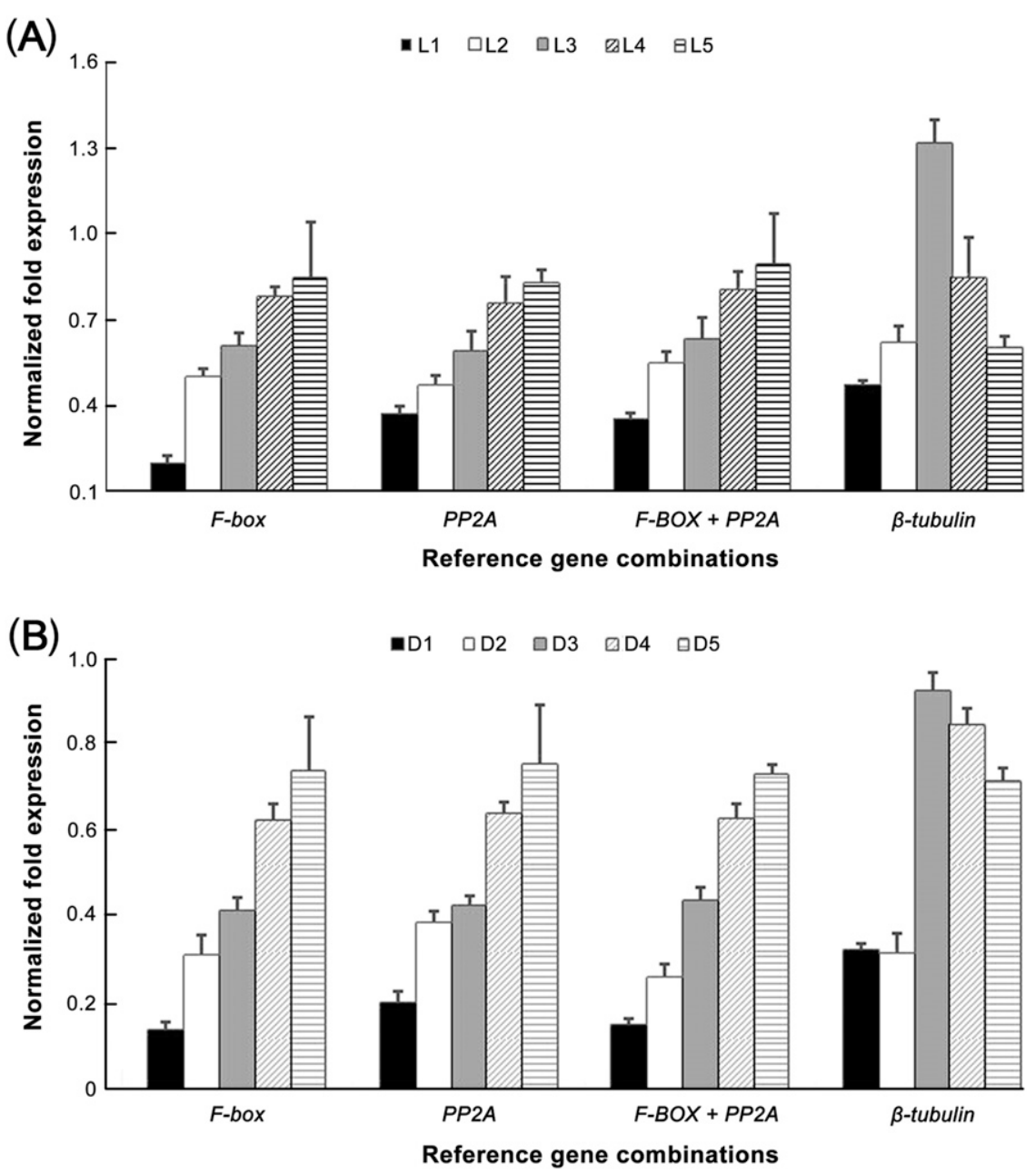

Fig. 4. The expression profiles of $\mathrm{CmF3H}$ at different floral developmental stages under illuminated and dark conditions. Normalization was performed using individual and combined reference genes: F-box, $P P 2 A$, $\beta$-tubulin, and F-box $+P P 2 A$. (A) Illuminated samples; (B) non-illuminated samples.

(Hu et al., 2009; Jian et al., 2008)], rice [Oryza sativa (Jain et al., 2006)], tomato (Løvdal and Lillo, 2009), cineraria (Jin et al., 2013), and tobacco [Nicotiana tabacum (Schmidt and Delaney, 2010)]. Actin was the last traditional reference gene selected in the present study, which has been used as a reliable reference gene for many years. However, further studies have revealed that it is not stable when experimental conditions change (Radonić et al., 2004). Therefore, it is necessary to evaluate the stability of this gene under specific experimental conditions. In our study, Actin performed well under different light conditions, which was similarly reported in soybean (Hu et al., 2009; Jian et al., 2008), but is contrary to the findings from studies in rice (Kim et al., 2003), sugarcane [Saccharum officinarum (Iskandar et al., 2004)], potato [Solanum tuberosum (Nicot et al., 2005)], and chamomile (Fu et al., 2013).

In general, the six new reference genes that we selected were more stable than the three conventional genes. In chrysanthemum, $C D P K$ yielded high $M$ values under different light conditions during the floral developmental process. Ullanat and Jayabaskaran (2002) found that in cucumber (Cucumis sativus), light differentially regulates $C D P K$ transcript levels in a tissue-specific manner. Therefore, whether $C D P K$ can perform better in the different

simple. Understanding the expression patterns of key genes involved in the anthocyanin biosynthetic pathway of chrysanthemum under various conditions will thus help us to understand the molecular mechanism of color generation in chrysanthemum. However, the molecular mechanism underlying this induction remains poorly understood. In chrysanthemum, Efl $\alpha$ and $P P 2 A$ have been shown to be stably expressed under different biotic and abiotic stresses ( $\mathrm{Gu}$ et al., 2011), but no detailed validation has been conducted to test their stability at different floral developmental stages and light conditions.

To our knowledge, this was the first systematic study to simultaneously investigate the expression stability of reference genes involved in flower color, floral development, and light effects in chrysanthemum. Based on the results of the present study, of the nine candidate genes, the traditional housekeeping gene $\beta$-tubulin yielded poor values in most of the sample sets, which was consistent with studies performed in cineraria (Jin et al., 2013), chinese iris (Gu et al., 2014), oil palm (Yeap et al., 2014), and cork oak [Quercus variabilis (Marum et al., 2012)]. Another conventional gene, $E F 1 \alpha$, was not very stably expressed in the present study, which was inconsistent with the studies in soybean [Glycine max tissues of chrysanthemum such as the tubular florets also requires further study. $P P 2 A$, which encodes the serine/threonine phosphatase, has been implicated in metabolism, DNA replication, transcription and translation, cell cycle, and signal transduction (Janssens and Goris, 2001). Some studies have found that $P P 2 A$ is stable and is therefore suitable for use as a reference gene (Artico et al., 2010; Løvdal and Lillo, 2009). Compared with $\beta$-tubulin and $E F 1 \alpha, P P 2 A$ was stably expressed to some extent in our study and was highly stable in different color lines but unstable under different light conditions at various floral developmental stages. This result is consistent with the results from the different flower color lines and floral developmental stages of cineraria (Jin et al., 2013). F-box, which plays important roles in the signal transduction of plant hormones and the biological processes of self-incompatibility and floral development (Craig and Tyers, 1999), was the most stable gene in this study. Liu et al. (2012) and Mafra et al. (2012) reported that $F$-box was the most stable reference gene in tobacco and citrus (Citrus sp.) under conditions of viral infections, results that are consistent with the present study. In the six new genes we selected, $E 3$ and $A B C$ had unstable expression in all sample sets, and the $\mathrm{M}$ value of $E 3$ remained especially high in all color lines. By contrast, TIP41 expressed constantly in all sample sets, which 
was consistent with the studies performed in tomato (ExpositoRodriguez et al., 2008) and arabidopsis (Czechowski et al., 2005), but contrary to a study performed in chamomile (Fu et al., 2013). Therefore, we should choose reference genes that are suitable for normalization according to different experimental conditions.

THE SUPERIORITY OF A COMPREHENSIVE ANALYSIS OF REFERENCE GENE EXPRESSION. Although RT-qPCR has been widely used over the past few years, normalization of reference genes still attracts some criticism. There is no consensus as to which type of algorithm should be used to determine the stability of gene expression because different statistical methods, which are based on different principles, yield potentially contradictory results from the same data. Several strategies have been introduced to normalize the expression of reference genes, including software such as geNorm (Vandesompele et al., 2002), NormFinder (Andersen et al., 2004), qBase plus (Hellemans et al., 2007), and BestKeeper (Pfaffl, 2001). Among the studies in which geNorm, NormFinder, and qBase plus have been simultaneously used for normalization, some minor differences have been shown among these three programs in terms of evaluating the best sets of reference genes in blueberry (Die and Rowland, 2013), oil palm (Yeap et al., 2014), and citrus (Yan et al., 2012). Moreover, studies in longan [Dimocarpus longan (Lin and Lai, 2010)], tobacco (Schmidt and Delaney, 2010), and rubber tree [Hevea brasiliensis ( $\mathrm{Li}$ et al., 2011)] have reported substantial differences between geNorm and NormFinder. The inconsistencies between these methods were expected because they are based on distinct statistical algorithms. In this article, all three methods were used to analyze the data to reduce the risk of inconsistent results. Although the ranking orders of gene stability generated by these methods were not exactly the same, the ranks of the most unstable genes were identical in all sample sets, which was similar to the results of a study in wheat [Triticum aestivum (Paolacci et al., 2009)]. In addition, the two most stable genes recommended by the three methods, F-box and $P P 2 A$, were the same in all of the samples.

THE RELIABILITY OF COMPREHENSIVE EVALUATION ON REFERENCE GENES. The expression pattern analysis of $\mathrm{CmF} 3 \mathrm{H}$ under different light conditions and developmental stages of the capitulum was further performed to validate the usefulness of the two most stable genes selected. When normalized by $F$-box, the relative expression level of $\mathrm{CmF3H}$ showed the same pattern as when using the combination of F-box and PP $2 A$, indicating that the two most stable genes are sufficient for RT-qPCR analysis of gene expression. In addition, Andersen and colleagues noted that geNorm may be insensitive to coregulated reference genes (Andersen et al., 2004). Because there are no coregulated reference genes in our study, the results calculated by geNorm are more convincing.

\section{Literature Cited}

Albert, N.W., D.H. Lewis, H. Zhang, L.J. Irving, P.E. Jameson, and K. Davies. 2009. Light-induced vegetative anthocyanin pigmentation in Petunia. J. Expt. Bot. 60:2191-2202.

Andersen, C.L., J.L. Jensen, and T.F. Ørntoft. 2004. Normalization of real-time quantitative reverse transcription-PCR data: A modelbased variance estimation approach to identify genes suited for normalization, applied to bladder and colon cancer data sets. Cancer Res. 64:5245-5250.

Anderson, N.O. 2006. Chrysanthemum (Chrysanthemum $\times$ grandiflora Tzvelv.), p. 389-437. In: Anderson, N.O. (ed.). Flower breeding and genetics issues, challenges and opportunities for the 21st century. Springer-Verlag, New York, NY.
Artico, S., S.M. Nardeli, O. Brilhante, M.F. Grossi-de-Sa, and M. AlvesFerreira. 2010. Identification and evaluation of new reference genes in Gossypium hirsutum for accurate normalization of real-time quantitative RT-PCR data. BMC Plant Biol. 10:49-60.

Brugliera, F., G.Q. Tao, U. Tems, G. Kalc, E. Mouradova, K. Price, K. Stevenson, N. Nakamura, I. Stacey, and Y. Katsumoto. 2013. Violet/blue chrysanthemums-metabolic engineering of the anthocyanin biosynthetic pathway results in novel petal colors. Plant Cell Physiol. 54:1696-1710.

Bustin, S.A. 2002. Quantification of mRNA using real-time reverse transcription PCR (RT-PCR): Trends and problems. J. Mol. Endocrinol. 29:23-29.

Chao, W.S., M. Dogramaci, M.E. Foley, D.P. Horvart, and J.V. Anderson. 2012. Selection and validation of endogenous reference genes for qRT-PCR analysis in leafy spurge (Euphorbia esula). PLoS One 7:e42839.

Coker, J.S. and E. Davies. 2003. Selection of candidate housekeeping controls in tomato plants using EST data. Biotechniques 35:740-749.

Craig, K.L. and M. Tyers. 1999. The F-box: A new motif for ubiquitin dependent proteolysis in cell cycle regulation and signal transduction. Prog. Biophys. Mol. Biol. 72:299-328.

Czechowski, T., M. Stitt, T. Altmann, M.K. Udvardi, and W.R. Scheible. 2005. Genome-wide identification and testing of superior reference genes for transcript normalization in Arabidopsis. Plant Physiol. 139:5-17.

Die, J.V. and L.J. Rowland. 2013. Superior cross-species reference genes: A blueberry case study. PLoS One 8:e73354.

Dong, Y.H., L. Beuning, K. Davies, D. Mitra, B. Morris, and A. Kootstra. 1998. Expression of pigmentation genes and photo-regulation of anthocyanin biosynthesis in developing Royal Gala apple flowers. Funct. Plant Biol. 25:245-252.

Exposito-Rodriguez, M., A.A. Borges, A. Borges-Perez, and J.A. Perez. 2008. Selection of internal control genes for quantitative realtime RT-PCR studies during tomato development process. BMC Plant Biol. 8:131.

Fu, J., Y. Wang, H. Huang, C. Zhang, and S. Dai. 2013. Reference gene selection for RT-qPCR analysis of Chrysanthemum lavandulifolium during its flowering stages. Mol. Breed. 31:205-215.

Gu, C., S. Chen, Z. Liu, H. Shan, H. Luo, Z. Guan, and F. Chen. 2011. Reference gene selection for quantitative real-time PCR in chrysanthemum subjected to biotic and abiotic stress. Mol. Biotechnol. 49:192-197.

Gu, C.S., L. Liu, C. Xu, Y. Zhao, X. Zhu, and S. Huang. 2014. Reference gene selection for quantitative real-time RT-PCR normalization in Iris lactea var. chinensis roots under cadmium, lead, and salt stress conditions. ScientificWorldJournal 21:1-7.

Gutierrez, L., M. Mauriat, S. Guénin, J. Pelloux, J.F. Lefebvre, R. Louvet, C. Rusterucci, T. Moritz, F. Guerineau, C. Bellini, and O. Van Wuytswinkel. 2008. The lack of a systematic validation of reference genes: A serious pitfall undervalued in reverse transcription-polymerase chain reaction (RT-PCR) analysis in plants. Plant Biotechnol. J. 6:609-618. Hellemans, J., G. Mortier, A.D. De Paepe, F. Speleman, and J. Vandesompele. 2007. qBase relative quantification framework and software for management and automated analysis of realtime quantitative PCR data. Genome Biol. 8:R19.

Higuchi, K., C. Fockler, G. Dollinger, and R. Watson. 1993. Kinetic PCR analysis: Real-time monitoring of DNA amplification reactions. Biotechnology 11:1026-1030.

Hu, R.B., C.M. Fan, H.Y. Li, Q.Z. Zhang, and Y.F. Fu. 2009. Evaluation of putative reference genes for gene expression normalization in soybean by quantitative real-time RT-PCR. BMC Mol. Biol. 10:93-104.

Huang, H., K. Hu, K. Han, Q. Xiang, and S. Dai. 2013. Flower colour modification of chrysanthemum by suppression of $F 3^{\prime} H$ and overexpression of the exogenous Senecio cruentus F3'5'H gene. PLoS One 8:e74395.

Huggett, J.F., K. Dheda, S.A. Bustin, and A. Zumla. 2005. Real-time RT-PCR normalisation; strategies and considerations. Genes Immun. 6:279-284. 
Iskandar, H.M., R.S. Simpson, R.E. Casu, G.D. Bonnett, D.J. Maclean, and J.M. Manners. 2004. Comparison of reference genes for quantitative real-time polymerase chain reaction analysis of gene expression in sugarcane. Plant Mol. Biol. Rpt. 22:325-337.

Jain, M., A. Nijhawan, A.K. Tyagi, and J.P. Khurana. 2006. Validation of housekeeping genes as internal control for studying gene expression in rice by quantitative real-time PCR. Biochem. Biophys. Res. Commun. 34:646-651.

Janssens, V. and J. Goris. 2001. Protein phosphatase 2A: A highly regulated family of serine/threonine phosphatases implicated in cell growth and signaling. Biochem. J. 353:417-439.

Jian, B., B. Liu, Y.R. Bi, W.S. Hou, C.X. Wu, and T.F. Han. 2008. Validation of internal control for gene expression study in soybean by quantitative real-time PCR. BMC Mol. Biol. 9:59-72.

Jin, X., J. Fu, S. Dai, Y. Sun, and Y. Hong. 2013. Reference gene selection for qPCR analysis in cineraria developing flowers. Sci. Hort. 153:64-70.

Kim, B.R., H.Y. Nam, S.U. Kim, S.I. Kim, and Y.J. Chang. 2003. Normalization of reverse transcription quantitative-PCR with housekeeping genes in rice. Biotechnol. Lett. 25:1869-1872.

Lee, J.M., J.R. Roche, D.J. Donaghy, A. Thrush, and P. Sathish. 2010. Validation of reference genes for quantitative RT-PCR studies of gene expression in perennial ryegrass (Lolium perenne L.). BMC Mol. Biol. 11:8-21.

Li, H.P., Y.X. Qin, X.H. Xiao, and C.R. Tang. 2011. Screening of valid reference genes for real-time RT-PCR data normalization in Hevea brasiliensis and expression validation of a sucrose transporter gene HbSUT3. Plant Sci. 181:132-139.

Li, Y., K. Mao, C. Zhao, X. Zhao, H. Zhang, H. Shu, and Y. Hao. 2012. MdCOP1 ubiquitin E3 ligases interact with $M d M Y B 1$ to regulate light-induced anthocyanin biosynthesis and red fruit coloration in apple. Plant Physiol. 160:1011-1022.

Lin, Y.L. and Z.X. Lai. 2010. Reference gene selection for qPCR analysis during somatic embryogenesis in longan tree. Plant Sci. 178:359-365.

Liu, D., L. Shi, C. Han, J. Yu, D. Li, and Y. Zhang. 2012. Validation of reference genes for gene expression studies in virus-infected Nicotiana benthamiana using quantitative real-time PCR. PLoS One 7:e46451.

Livak, K.J. and T.D. Schmittgen. 2001. Analysis of relative gene expression data using real-time quantitative PCR and the $2^{-\Delta \Delta C T}$ method. Methods 25:402-408.

Løvdal, T. and C. Lillo. 2009. Reference gene selection for quantitative real-time PCR normalization in tomato subjected to nitrogen, cold, and light stress. Anal. Biochem. 387:238-242.

Mafra, V., K.S. Kubo, M. Alves-Ferreira, M. Ribeiro-Alves, R.M. Stuart, L.P. Boava, C.M. Rodrigues, and M.A. Machado. 2012. Reference genes for accurate transcript normalization in citrus genotypes under different experimental conditions. PLoS One 7:e31263.

Martin, J.A. and Z. Wang. 2011. Next-generation transcriptome assembly. Nat. Rev. Genet. 12:671-682.

Marum, L., A. Miguel, C.P. Ricardo, and C. Miguel. 2012. Reference gene selection for quantitative real-time PCR normalization in Quercus suber. PLoS One 7:e35113.

Mortazavi, A., B.A. Williams, K. McCue, L. Schaeffer, and B. Wold. 2008. Mapping and quantifying mammalian transcriptomes by RNASeq. Nat. Methods 5:621-628.

Nicot, N., J.F. Hausman, L. Hoffmann, and D. Evers. 2005. Housekeeping gene selection for real-time RT-PCR normalization in potato during biotic and abiotic stress. J. Expt. Bot. 56:2907-2914.

Noda, N., R. Aida, S. Kishimoto, K. Ishiguro, M. Fukuchi-Mizutani, Y. Tanaka, and A. Ohmiya. 2013. Genetic engineering of novel bluer-colored chrysanthemums produced by accumulation of delphinidin-based anthocyanins. Plant Cell Physiol. 54:1684-1695.

Nolan, T., R.E. Hands, and S.A. Bustin. 2006. Quantification of mRNA using real-time RT-PCR. Nat. Protoc. 1:1559-1582.

Paolacci, A.R., O.A. Tanzarella, E. Porceddu, and M. Ciaffi. 2009. Identification and validation of reference genes for quantitative RTPCR normalization in wheat. BMC Mol. Biol. 10:11-37.

Pfaffl, M.W. 2001. A new mathematical model for relative quantification in real-time RT-PCR. Nucleic Acids Res. 29:e45.

Radonić, A., S. Thulke, I.M. Mackay, O. Landt, W. Siegert, and A. Nitsche. 2004. Guideline to reference gene selection for quantitative real-time PCR. Biochem. Biophys. Res. Commun. 313:856-862.

Rozen, S. and H. Skaletsky. 1999. Primer3 on the WWW for general users and for biologist programmers, p. 365-386Krawetz, S. and $\mathrm{S}$. Misener (eds.). Bioinformatics methods and protocols in the lines methods in molecular biology. Humana Press, Totowa, NJ.

Schmidt, G.W. and S.K. Delaney. 2010. Stable internal reference genes for normalization of real-time RT-PCR in tobacco (Nicotiana tabacum) during development and abiotic stress. Mol. Genet. Genomics 283:233-241.

Sun, W., C. Li, L. Wang, and S. Dai. 2010. Analysis of anthocyanins and flavones in different-colored flowers of chrysanthemum. Chinese Bul. Bot. 45:327-336 (abstr.).

Tanaka, Y. and A. Ohmiya. 2008. Seeing is believing: Engineering anthocyanin and carotenoid biosynthetic pathways. Curr. Opin. Biotechnol. 19:190-197.

Ullanat, R. and C. Jayabaskaran. 2002. Distinct light, cytokinin and tissue specific regulation of calcium dependent protein kinase gene expression in cucumber (Cucumis sativus). Plant Sci. 162:153-163.

Vandesompele, J., K. De Preter, F. Pattyn, B. Poppe, N. Van Roy, A. De Paepe, and F. Speleman. 2002. Accurate normalization of real-time quantitative RT-PCR data by geometric averaging of multiple internal control genes. Genome Biol. 3:1-11.

Wang, T., R. Hao, H. Pan, T. Cheng, and Q. Zhang. 2014. Selection of suitable reference genes for quantitative real-time polymerase chain reaction in Prunus mume during flowering stages and under different abiotic stress conditions. J. Amer. Soc. Hort. Sci. 139:113-122.

$\mathrm{Xu}$, Z.R. and Y.H. Li. 2006. Expression of the genes involved in anthocyanin biosynthesis of 'Tsuda' turnip. J. Plant Physiol. Mol. Biol. 32:583-586.

Yan, J., F. Yuan, G. Long, L. Qin, and Z. Deng. 2012. Selection of reference genes for quantitative real-time RT-PCR analysis in citrus. Mol. Biol. Rpt. 39:1831-1838.

Yeap, W.C., J.M. Loo, Y.C. Wong, and H. Kulaveerasingam. 2014. Evaluation of suitable reference genes for qRT-PCR gene expression normalization in reproductive, vegetative tissues and during fruit development in oil palm. Plant Cell Tissue Organ Cult. 116:55-66.

Yu, B., D. Zhang, C. Huang, M. Qian, X. Zheng, Y. Teng, J. Su, and Q. Shu. 2012. Isolation of anthocyanin biosynthetic genes in red chinese sand pear (Pyrus pyrifolia Nakai) and their expression as affected by organ/tissue, cultivar, bagging and fruit side. Sci. Hort. 136:29-37.

Zhao, W., J. Zhao, L. He, Y. Sun, and H. Cai. 2013. Molecular structure and the second introns variation of gene $F 3^{\prime} H$ of two medicinal Chrysanthemum morifolium populations. Biochem. Syst. Ecol. 51:251-258.

Zheng, Y., J.H. Li, H.P. Xin, N. Wang, L. Guan, B.H. Wu, and S.H. Li. 2013. Anthocyanin profile and gene expression in berry skin of two red Vitis vinifera grape cultivars that are sunlight dependent versus sunlight independent. Austral. J. Grape Wine Res. 19:238-248.

Zhou, B. 2004. Expression of genes related to anthocyanin biosynthesis in strawberry fruits under light and dark conditions. Master's diss., Northeast Forestry Univ., Harbin, China. 
Supplemental Table 1. List of samples used for gene expression studies in Chrysanthemum $\times$ morifolium 'Reagan'.

\begin{tabular}{rrrl}
\hline & & Floral developmental & \\
No. & Flower color & stage & \multicolumn{1}{c}{ Light treatment } \\
\hline 1 & Purple & S1 & Illuminated condition \\
2 & Purple & S2 & Illuminated condition \\
3 & Purple & S3 & Illuminated condition \\
4 & Purple & S4 & Illuminated condition \\
5 & Purple & S5 & Illuminated condition \\
6 & Purple & S1 & Non-illuminated condition \\
7 & Purple & S2 & Non-illuminated condition \\
8 & Purple & S3 & Non-illuminated condition \\
9 & Purple & S4 & Non-illuminated condition \\
10 & Purple & S5 & Non-illuminated condition \\
11 & Red & S1 & Illuminated condition \\
12 & Red & S2 & Illuminated condition \\
13 & Red & S3 & Illuminated condition \\
14 & Red & S4 & Illuminated condition \\
15 & Red & S5 & Illuminated condition \\
16 & Red & S1 & Non-illuminated condition \\
17 & Red & S2 & Non-illuminated condition \\
18 & Red & S3 & Non-illuminated condition \\
19 & Red & S4 & Non-illuminated condition \\
20 & Red & S5 & Non-illuminated condition \\
21 & Pink & S1 & Illuminated condition \\
22 & Pink & S2 & Illuminated condition \\
23 & Pink & S3 & Illuminated condition \\
24 & Pink & S4 & Illuminated condition \\
25 & Pink & S5 & Illuminated condition \\
26 & Pink & S1 & Non-illuminated condition \\
27 & Pink & S2 & Non-illuminated condition \\
28 & Pink & S3 & Non-illuminated condition \\
29 & Pink & S4 & Non-illuminated condition \\
30 & Pink & S5 & Non-illuminated condition \\
\hline & & &
\end{tabular}

